in principle can be estimated from measurements of anisotropies in the cosmic background radiation) are found to be greater than predicted by these special geometries.

This is certainly a controversial viewpoint; apart from the questions it raises philosophically, it needs technical development to be made precise enough for verification. In fact, it eventually leads to the major unsolved problem of a probability measure on the initial conditions for the Universe, without which all this kind of speculation is on shaky ground.

Marek Abramowicz and George Ellis are at the International School for Advanced Studies, 34014 Trieste, Italy.

\title{
Palaeoclimate
}

\section{Finer grains of truth}

\section{Peter D. Moore}

Pollen analysis of organic peats and lake sediments is used extensively to reconstruct past changes in vegetation and climate. But such studies have concentrated mainly on changes detectable on a timescale of centuries or even millenia. Changes over timescales of a few decades or less are usually considered too fine to be resolved by the technique. But several recent projects show that this is not always so: Green and co-workers ${ }^{1.2}$ for example, not only show that fine-resolution analysis is possible, but also relate it to environmental variables such as short-term climatic fluctuations and fire frequency.

Several problems stand in the way of fine-resolution pollen analysis. In many lakes, sediment is resuspended and redeposited during periods of turbulence ${ }^{3}$ : it can even be 'focused' into certain parts of the basin ${ }^{4}$. These processes mix materials over a period of years and, although the general trends in the fossil record may be preserved, short-term changes are masked and smoothed. In peat deposits, the main problem is one of pollen migration within the loosely packed upper layers of the growing peat mass. Experiments in which pollen is added to columns of peat suggest that vertical transport with water flow limits the resolution potential of the analysis ${ }^{5}$.

Despite these concerns, some studies both on lake sites and in peat deposits do permit fine-scale resolution. In deep, steep-sided lakes, resuspension may not be significant and sediments can even develop annual laminations, demonstrating the stability of sedimentation. In peats, several studies involving close sampling, even in poorly compacted layers, show a pollen stratification that argues against vertical pollen migration. Van Geel and Middeldorp ${ }^{6}$ have now analysed the stratigraphy of freshly formed peat in Carbury Bog, Ireland, which shows strong peaks in pollen from Ericaceae, Gramineae and Pinus in the top $10 \mathrm{~cm}$ of unconsolidated peat, formed over the past 10 years. If there were significant downwash of pollen through the upper peat layers, these peaks would have been lost.
Green and colleagues have developed techniques of comparing such fine-scale pollen changes with fluctuations in environmental factors in peat ${ }^{1}$ and in lake $^{2}$ sites. By looking at the sequential records of pollen, charcoal and (independently obtained) rainfall data as time series, they can cross-correlate the data sets and also allow for lag times between the causative factor and its response in the pollen record. They find that certain pollen taxa, Eucalyptus, grasses and Compositae (Tubuliflorae), show a strong positive response to rainfall, and that even small variations in rainfall are reflected in the pollen stratigraphy. These are also correlations with charcoal deposition. Eucalyptus, for example, responds positively with a 5-10-year lag phase, suggesting that trees damaged by fire had recovered rather than being destroyed.

But the relationship between pollen and charcoal is complicated by problems of soil erosion and consequent enhanced pollen input following a fire. And the origins of the charcoal itself must be considered: Clark $^{7}$ has examined charcoal transport in relation to particle size and considers that fine particles (such as those found on microscope slides for pollen preparations) are transported over long distances and do not necessarily reflect fires in the immediate catchment. So correlations between pollen and charcoal must take into account the dispersal characteristics of both pollen grains and carbon particles.

These recent studies indicate that the technique of pollen analysis, both of peats and certain types of lake sediments, does have potential in providing fine-resolution information concerning vegetation changes and hence the variation of other environmental factors in time.

1. Green, D. et al. J. Ecol. 76, 790-806 (1988).

2. Green, D. \& Dolman, G.S.J. Biogeogr. 15, 685-701 (1988)

3. Davis, M.B. Science 162, 796-799 (1967).

4. Davis, M.B. \& Ford, M.S. Limnol. Oceanogr. 27, 137 (1982).

5. Clymo, R.S. \& Mackay, D. New Phytol. 105, 175 (1987).

6. Van Geel, B. \& Middeldorp, A.A. New Phytol. 109, 377392 (1988).

7. Clark, J.S. Quat. Res. 30, 67-80 (1988).

Peter D. Moore is Reader in Ecology in the Division of Biosphere Sciences, King's College, Campden Hill Road, London W8 7AH, UK.

\section{Daedalus}

\section{Diamonds forever}

BRITTLE fracture is the ultimate engineering catastrophe. Microscopic cracks lurk in all solid surfaces; they gape slightly under load, and may even extend a little. Sooner or later the component may meet a stress great enough for a crack to run away, like a cloth tearing in two from a nick in one edge, and disaster strikes.

Now Daedalus has the answer. When a microcrack forms or extends, the local chemical bonds are pulled apart, leaving two pure free-radical surfaces. Such reactive faces must combine almost instantly with local air and water. But, says Daedalus, suppose the atmosphere around the crack were not air, but a polymerizable gas such as ethylene? Each radical-covered surface would be a splendid heterogeneous polymerization catalyst: the crack would be instantly bonded with polyethylene.

But polyethylene is not really an ideal 'glue'. Daedalus recalls that methanehydrogen mixtures can deposit a tenaciously bonded diamond film on surfaces hot enough to decompose the methane to methyl radicals. A fresh crack surface dense with free radicals should do this even in the cold, by direct electron-transfer. A crack formed in a methane-hydrogen atmosphere should be instantly sealed with strong, stiff, inert, non-melting diamond: the finest imaginable engineering glue.

So Daedalus plans to 'crack-proof' industrial structures such as cranes, bridges and pylons, by slowly stressing them up to working load in an atmosphere of methane-hydrogen. As each microcrack in the structure opened up, it would be automatically sealed with diamond. When the structure finally reached its design load, each stressed part would be studded with tiny diamond inclusions: closing all its potentially deadly little cracks, and prestressing it perfectly to its service duty. Even structures like airframes, plagued by the growth of fatigue cracks in service, might be 'immunized' against them by the sealing of the sites from which they begin.

And brittle materials like ceramics could at last be tamed. A ceramic turbine blade, gradually loaded in hot methane-hydrogen, would show its true strength at last. Deprived of the surface flaws from which to fail by brittle fracture, it could be loaded almost up to the point of plastic flow. Thus the splendid corrosion resistance and hightemperature performance of ceramics will be made safely accessible to engineers. All the heat-stressed valves, blades, pistons and cams of modern high-power engines, so vulnerable even in metal, will be safe and durable at last. Even existing engines and turbines might be crack-proofed by pumping them up to speed with hot methane-hydrogen.
David Jones 